Instituto Internacional de Investigación y Desarrollo Tecnológico Educativo INDTEC, C.A.

DOI: https://doi.org/10.29394/Scientific.issn.2542-2987.2020.5.17.9.184-204

OAI-PMH: http://www.indteca.com/ojs/index.php/Revista Scientific/oai

Ensayo Original / Original Essay

\title{
Estado del arte para la construcción de una Didáctica de la Historia Reciente
}

\author{
Autor: Alexis Antonio González Natera \\ Universidad Latinoamericana y del Caribe, ULAC \\ alangperiodista17@gmail.com \\ Caracas, Venezuela \\ https://orcid.org/0000-0003-1706-4054
}

\section{Resumen}

La presente producción intelectual constituye un recorrido por el Estado del Arte sobre tesis doctorales cuyo foco de atención ha sido la Didáctica, la Historia Reciente como disciplina historiográfica, y el análisis de las Caricaturas Políticas con fines distintos a la opinión periodística o el interés artístico. El propósito de la investigación es explorar, a modo de antecedentes, los aportes de estos trabajos para el desarrollo de una Didáctica de la Historia Reciente, como constructo dialéctico-interpretativo de las caricaturas políticas de Zapata, título bajo el cual se inscribe un trabajo doctoral de propia autoría, actualmente en desarrollo. El método utilizado corresponde a las técnicas de investigación documental. El corolario de este ensayo refleja las principales contribuciones recogidas en los documentos bajo escrutinio, en donde destacan la necesidad de incentivar la creatividad del docente y los alumnos en su labor didáctica, la importancia del currículo como base de las prácticas educativas, los problemas de aprendizaje de la Historia, el valor de la memoria en el abordaje de la Historia Reciente, y por último, el creciente interés del estudio de las caricaturas políticas con fines historiográficos.

Palabras clave: ciencias de la educación; modelo educacional; proceso de aprendizaje; método de enseñanza; historiografía.

Cómo citar este ensayo:

González, A. (2020). Estado del arte para la construcción de una Didáctica de la Historia Reciente. Revista Scientific, 5(17), 184-204, e-ISSN: 2542-2987. Recuperado de: https://doi.org/10.29394/Scientific.issn.2542-2987.2020.5.17.9.184-204

Fecha de Recepción: 02-03-2020
Fecha de Aceptación: 16-06-2020
Fecha de Publicación: 05-08-2020 


\title{
State of the art for the construction of a Teaching of Recent History
}

\begin{abstract}
The present intellectual production constitutes a journey through the State of Art on doctoral theses whose focus has been Didactics, Recent History as a historiographic discipline, and the analysis of Political Caricatures for purposes other than journalistic opinion or artistic interest. The purpose of the research is to explore, by way of background, the contributions of these works for the development of a Teaching of Recent History, as a dialecticalinterpretive construct of Zapata's political cartoons, a title under which a doctoral work is inscribed own authorship, currently under development. The method used corresponds to the documentary investigation techniques. The corollary of this essay reflects the main contributions collected in the documents under scrutiny, where they highlight the need to encourage the creativity of teachers and students in their didactic work, the importance of the curriculum as a basis for educational practices, learning problems of History, the value of memory in the approach to Recent History, and finally, the growing interest in the study of political cartoons for historiographical purposes.
\end{abstract}

Keywords: educational sciences; educational model; learning processes; teaching method; historiography.


https://doi.org/10.29394/Scientific.issn.2542-2987.2020.5.17.9.184-204
Date Received: 02-03-2020
Date Acceptance:

16-06-2020
Date Publication:

05-08-2020 


\section{Introducción}

La presente producción académica es parte integrante -a manera de antecedentes de investigación-, en la elaboración de una tesis doctoral de propia autoría titulada Didáctica de la Historia Reciente como constructo dialéctico-interpretativo de las caricaturas políticas de Zapata, cuyo propósito principal es la construcción de ésta Didáctica específica, a partir del análisis e interpretación de las caricaturas políticas del reconocido humorista y caricaturista venezolano, Pedro León Zapata (1929-2015).

Desde el inicio de este esfuerzo investigativo, se ha considerado que más allá de levantar el andamiaje teórico-metodológico que sirva de soporte-, es requisito indispensable conocer los avances que sobre la materia han adelantado otros investigadores, particularmente en aquellos trabajos validados como tesis doctorales en las áreas de interés. Tal es la intención de este escudriñamiento del estado del arte, realizado a partir de la revisión y análisis de cinco trabajos doctorales, recopilados mediante técnicas de investigación documental, según la cual la información necesaria está contenida en documentos que, en su amplio sentido como señalan Cázares, Christen, Jaramillo, Villaseñor y Zamudio (1990), incluye:

Todo material de índole permanente, es decir, al que se puede acudir como fuente o referencia en cualquier momento o lugar, sin que se altere su naturaleza o sentido, para que aporte información o rinda cuentas de una realidad o acontecimiento (pág. 18).

En consecuencia, el material bajo escrutinio aporta conocimientos sustantivos en torno al tema de nuestro interés, a contenidos específicos y los métodos utilizados en los mismos, que resultan de provecho para la propia investigación, y así satisfacer los requerimientos exigidos en el programa doctoral en curso, además de servir al propósito principal de este ensayo, el cual consiste en explorar, a modo de antecedentes, las contribuciones de estos 
escritos para el desarrollo ulterior de la tesis doctoral ya mencionada.

Es pertinente considerar como marco de referencia del presente trabajo, la construcción de una Didáctica específica aplicable a la disciplina historiográfica denominada Historia Reciente, que tal como indican Figueroa e Iñigo en su artículo Reflexiones para una definición de la Historia Reciente, contenido en la compilación que presentan Rajiad, Figueroa y López (2010): es vista como un campo en construcción y epistemológicamente inestable.

Este argumento permite además acercarnos a lo indicado por Caldera (2020a): quien aborda el concepto de didáctica desde una perspectiva epistemológica, al nombrar su empleo por parte de Wolfgang en Principios de la didáctica del año 1929. Allí se indica que el término giraba en torno al sentido y la necesidad de enseñar.

Por ello en su interpretación, Caldera (2020b), asegura que quienes se dedican al oficio de enseñar, se mueven entre dos elementos en común: "[...] un sentido lógico de querer aumentar el conocimiento y la necesidad de que sus discípulos aprendan, para ello utilizan todo un andamiaje de métodos, técnicas y recursos científicos, artísticos y de oficios" (pág. 389). A modo de justificación, los argumentos esgrimidos invitan a mantener una mente amplia, acuciosa y atenta a lo largo de este camino para develar el conocimiento requerido.

\section{Desarrollo}

\subsection{Didáctica universitaria: del currículo a las prácticas de enseñanza}

En este trabajo se aborda en forma paralela y complementaria algunas áreas fundamentales en educación, a decir de Giraldo (2012a): "[...] con el propósito principal de analizar, comprender e interpretar las relaciones que se tejen entre éstas; específicamente, entre el currículo de Medicina Veterinaria de la Universidad de Antioquia y las prácticas de enseñanza que despliegan sus profesores [...]" (pág. 19). La tesis de la autora constituye una 
investigación a través de la cual Giraldo (2012b), pretende:

[...] Comprender el currículo como una propuesta educativa que es interpretada y llevada a la práctica, concretamente al espacio áulico por los profesores; de tal forma, que el currículo en la clase se hace realidad gracias a la interacción entre docentes y estudiantes (pág. 19).

Para la autora, su foco de atención gira en torno al rol que cumple el docente como parte de los procesos de transformación curricular, reconociendo la presencia de las diversas variables que intervienen, las cuales no pueden ser desechadas en el análisis, dada a su importancia. No obstante, Giraldo (2012c), ratifica que se trata de: "[...] destacar que las ideas y las acciones de los profesores son fundamentales para asegurar el cambio educativo implícito en las transformaciones curriculares" (pág. 19).

El contenido revelado en este informe final por la autora, nos obliga a tener en cuenta que nuestra pretendida construcción didáctica, debe estar sostenida sobre la base de una sólida propuesta curricular, que permita dar viabilidad a la inserción del estudio de la Historia Reciente en la formación universitaria -quizá como cátedra electiva o de conocimiento general-, en carreras tales como Comunicación Social, Sociología, Trabajo Social, Educación u otras vinculadas a las Ciencias Humanísticas, Sociales o de la Educación, entendiendo que el currículo, como lo reconoce Caldera (2020c): "ofrece una gama de propósitos u objetivos, métodos, estrategias, actividades tomando como referencia los niveles y modalidades, los aspectos científicos, artísticos, profesionales y de oficios [...]" (pág. 386); convirtiéndolo en el material de apoyo más importante con que cuenta el docente.

\subsection{Creatividad del docente universitario}

Conforme explica la autora, su tesis deviene de la identificación y abordaje de un problema observado en la Universidad Autónoma de 
Zacatecas (UAZ), la cual estaba sumergida en un proceso de cambios orientados a la modernización de ciertas áreas. Interpretando a Ortega (2014a), estas labores implicarían un conjunto de iniciativas: "[...] con contenidos educativos y estrategias innovadoras en la docencia [...]" (pág. 39); coadyuvando así su promoción: "[...] hacia la internacionalización, afianzando el compromiso de la educación sin fronteras [...]" (pág. 39); además de ofrecer opciones de calidad para la ciudadanía.

Con referencia al detalle del trabajo revisado, la autora considera -en cuanto a la problematización de su estudio-, que aun cuando la organización tiene un programa de atención para la formación del docente, este es insuficiente. Más adelante, Ortega (2014b), dice que el mismo: "no especifica la conceptuación, rol, metodología, didáctica y pedagogía de esta formación" (pág. 39); afirma que la entidad: "no tiene un enfoque centrado en la creatividad desde su conceptuación, utilización, didáctica y evaluación" (pág. 39); asimismo, la autora recalca que: "no existe una amplia información para el docente en lo que se refiere a la evaluación de los aprendizajes del alumno y a cómo promover el desarrollo de la creatividad en ellos, en el marco de su formación" (pág. 39).

Tales señalamientos hacen que este trabajo, sea pertinente con la línea de investigación sobre Fundamentos y gestión de la enseñanza y el aprendizaje, establecida en el programa doctoral que actualmente estoy cursando, el cual constituye el eje orientador de la tesis a desarrollar. En consecuencia, es asimilable a nuestro interés, dada la premisa planteada, para resolver los problemas detectados en el proceso de enseñanza-aprendizaje de la Historia (u otras áreas del conocimiento), las soluciones apuntan a la necesidad lograr mayor motivación por parte del estudiante, haciendo que estos adopten un rol más activo dentro de esa dinámica. Adicionalmente se debe apoyar en un cambio de actitud por parte del docente, bajo el criterio de que éste debe asumir el papel de mediador, entendiendo la mediación, 
conforme a lo expresado por Ríos (2006), como:

Una experiencia de aprendizaje donde un agente mediador (padres, educadores), actúan como apoyo y se interponen entre el aprendiz y su entorno para ayudarle a organizar y a desarrollar su sistema de pensamiento y facilitar así la aplicación de nuevos instrumentos intelectuales a los problemas que se le presenten (pág. 406).

Asumir tales recomendaciones, resulta necesario para quienes decidimos emprender el exigente camino del estudio y compresión del mundo que nos rodea, analizar sus problemas y buscar respuestas a las cuestiones planteadas, dentro de un ambiente que, en la lógica de la posmodernidad, se fija como un escenario fragmentado, difuso, complejo, incierto y en ocasiones conflictivo, dentro del cual la mirada amplia, el pensamiento crítico, la intuición y en especial la creatividad son herramientas clave para lograr el éxito en ésta labor de reflexión profunda sobre determinados aspectos de esa realidad, más aun cuando se trata del campo educativo.

Siguiendo la línea de pensamiento planteada en su investigación, Ortega (2014c), reitera: "[...] la conveniencia de la formación del docente universitario en general y en didáctica de la creatividad en particular [...]" (pág. 39); dirigida hacia los profesores de la universidad en referencia. Tal consideración se sostiene en su propia experiencia como investigadora, pues ella aclara haber observado varias dificultades derivadas de las limitaciones en la formación de los docentes universitarios.

Con base en este estudio, se vislumbra varios aspectos a reflexionar en la construcción didáctica en desarrollo, y en su posterior aplicación. Entre estos aportes resulta destacable que, en el diseño, se tenga en cuenta las necesidades de los protagonistas del proceso de enseñanza aprendizaje docentes y alumnos-, llamado que es reiterativo en varios estudios y autores consultados. De igual forma, resultan de interés los razonamientos de la autora, con relación a la importancia de la creatividad. Al respecto, Ortega 
(2014d), enfatiza sobre: "[...] el valor teórico de la comprensión de la creatividad y su amplia relación con la formación didáctica del docente, asociada a la utilidad metodológica para ayudar a la resolución de problemas emergentes relacionados con su quehacer" (pág. 39).

Esta capacidad -propia del ser humano-, puede llegar a ser una herramienta de alto valor en la dinámica educativa, pues implica el potencial de generación de nuevas ideas, de nuevos conceptos y de relaciones entre estos, además de otros atributos que la caracterizan, y que con la práctica se pueden cosechar. De hecho, así lo ratifica Ortega (2014e), la creatividad: "[...] requiere ser practicada para convertirla en habilidad y a partir de ahí reconocerla, reflexionarla, compartirla, practicarla, educarla y desarrollarla para la formación integral" (pág. 42); además, en su estudio aborda la vinculación entre la creatividad y la didáctica, pues considera que la primera debe estar presente en todas las fases del proceso educativo, incluso desde la formación del docente.

De las conclusiones alcanzadas por Ortega (2014f), se rescata el hecho de que los profesionales participantes en el estudio: "no se conciben como docentes creativos y desconocen la forma de evaluar la creatividad en sus alumnos" (pág. 41); tal resultado, la motiva a reiterar que: "la formación docente, la pedagogía, la didáctica y la creatividad, requieren espacios concretos para análisis, reflexión y aplicación en todo el proceso de enseñanza aprendizaje [...]" (pág. 42); aludiendo en particular a la casa de estudio en donde realizó su investigación.

\subsection{La historia que se aprende en la escuela}

En su introducción, la autora comienza reconociendo las dificultades que implica realizar trabajos de investigación sobre el área educativa en Venezuela, y aún más si estos, como alega Millán (2008a), son abordados: "[...] en campo de la Didáctica de las Ciencias Sociales y de las nuevas 
epistemologías y metodologías, que incipientemente son impulsadas por algunos investigadores [...]" (pág. 17); mencionando entre ellos a Joaquim Prats, cuyas investigaciones también forman parte de la revisión documental en nuestro planteamiento del problema de tesis doctoral.

Dichos trabajos tendrían la intención de arrojar luces con relación a los problemas detectados en el ámbito del aprendizaje de la Historia en la educación secundaria. No obstante, la parálisis de las investigaciones en este sector, a decir de Millán (2008b), respondería acaso al: "[...] interés por estudiar y solucionar otros problemas educativos vinculados a aspectos económicos, presupuestarios o éticos [...]" (pág. 17); en el país.

En virtud, Millán (2008c), enfatiza sobre aspectos de su interés en el desarrollo de la investigación, como son:

[...] La percepción y el conocimiento de la historia que se aprende en la Escuela Básica desde la perspectiva del alumnado, y por la casi inexistencia de investigaciones de este tipo en Venezuela, que tengan al alumno como centro y protagonista de los aprendizajes de esta disciplina escolar [...] (pág. 17).

Esto justifica la realización de su tesis doctoral, argumentando estar consciente de su importancia en el campo de la Didáctica de las Ciencias Sociales, y en la disciplina didáctica de la Historia, por lo novedoso del tema.

La revisión a profundidad del trabajo de esta autora se me hace pertinente, pues se aprecian muchos puntos coincidentes con relación a las dificultades detectadas en el proceso de enseñanza-aprendizaje de la Historia, a la crítica hacia los métodos tradicionales empleados, y a la necesidad de brindar especial atención a la perspectiva del alumnado, además de lo novedoso del tema en estudio, tanto más si es abordado dentro el contexto venezolano, en donde se han hallado evidentes carencias en cuanto a la producción de investigaciones sobre la materia.

Por otra parte, suscribo que la contribución de estudios de esta 
naturaleza, pues como indica Millán (2008d), será de suma importancia: “[...] para el campo de la didáctica como ciencia en consolidación [...]" (pág. 18); teniendo en cuenta el valor que ella le atribuye a la perspectiva del alumno dentro del proceso educativo, a partir del conocimiento que se tiene sobre las dificultades implícitas en el mismo, las cuales se confirman en distintos estudios adelantados al respecto.

\subsection{Historia reciente: ¿exilio, migración o destierro?}

Este trabajo se inscribe en el ámbito de la denominada Historia Reciente -ya mencionada en este ensayo-, concretamente vista en el territorio argentino, en donde Gatica (2010a), aborda: "[...] el análisis de experiencias exiliares de trabajadores chilenos que se radicaron en el Noreste de la Provincia de Chubut, en Patagonia Argentina [...]" (pág. 4); luego del golpe de estado contra el presidente Salvador Allende, en septiembre del año 1973.

Paralelamente, al momento de plantear la fundamentación teórica para incorporarse en el Doctorado en Historia, Gatica (2010b), destaca que quiso abocarse a problematizar: "[...] un tema que permitiera relacionar los contenidos curriculares del espacio docente en que me desempeño como Profesora Asociada, la Historia de América Latina Contemporánea; con lo que ha sido mi trayectoria en investigación [...]" (pág. 4); a la cual se habría dedicado durante más de veinte años, vale decir, la historia de la clase obrera en la referida región.

La tarea emprendida por esta investigadora también está inscrita en la necesidad de aportar el instrumental teórico, metodológico, e investigaciones fácticas que resulten incluyentes, y que permitan un análisis en profundidad de la sociedad con la que interactúa cotidianamente. Por tal motivo, Gatica (2010c), asevera estar: "convencida que Historia, Identidad, y Memoria son problemáticas no sólo relevantes para la vida académica, sino también para vivir nuestro presente y nuestro futuro" (pág. 4); Igualmente, Jelín (2002), 
citada por Gatica (2010d), sostiene que el historiador: "puede tornarse agente público, y sus posturas pueden tener consecuencias políticas que van más allá de los saberes disciplinarios, y los debates académicos" (pág. 4).

Estas reflexiones, son aspectos relevantes en nuestra propia investigación a propósito de inducir, a través de la didáctica propuesta, la proactividad y creatividad de docentes y alumnos, en el proceso de enseñanza aprendizaje de la Historia Reciente -particularmente la referida al caso venezolano de los últimos años-, en donde se tendrá un primer contacto con la realidad histórica mediante las caricaturas políticas, como representación de un momento y espacio concreto, la cual tendrá que ser indagada, contextualizada y analizada con el apoyo de otros recursos documentales e incluso testimoniales, tal como se desprende de lo expuesto por la autora.

Esta metodología o práctica, además involucra una dimensión personal, subjetiva y afectiva, que supone para Gatica (2010e): "una constante relación entre los sujetos que participan, lo que implica una cualitativa diferencia en relación a las fuentes más tradicionales" (págs. 4-5); así como la factibilidad de la investigación, dados los escasos repositorios documentales, la llevó a optar por trabajar con historia oral, es decir, el testimonio de las personas que fueron víctimas o testigos de la situación.

Si bien manifiesta no desconocer el valor de la estadística, ni menospreciar su empleo, Gatica (2010f), eligió de preferencia: “[...] nombrar y conocer a quienes han compartido con nosotros vivencias y experiencias [...]" (pág. 5); explicitando con ello su propia "[...] inscripción en la construcción de la información documental, no objetivándonos" (pág. 5); como ocurre en la práctica historiográfica tradicional. Ello reafirma la importancia del contacto testimonial y de la memoria individual y colectiva, en la indagación de la Historia Reciente.

El trabajo desarrollado, en palabras de Gatica (2010g): "se halla en la encrucijada entre la historia del pasado reciente y la memoria; con una 
perspectiva "desde abajo", buscando conjugar la no uniformidad de la experiencia humana, con la pretensión de generalidad y explicación de la ciencia social" (pág. 5).

De acuerdo con esto, Gatica (2010h), expone que las narraciones o testimonios obtenidos fueron revisados: "[...] procurando develar las representaciones que subyacen, y comprendiendo el modo en que actuaron" (pág. 5); por lo cual la autora acota que "las memorias tienen un carácter subjetivo y en permanente transformación [...]" (pág. 5); haciéndolas singulares -coincidiendo así con las apreciaciones o juicios de varios expertos en la materia.

No obstante, Gatica (2010i), como historiadora, recuerda que debe: "[...] inscribirlas en un contexto histórico global, para procurar esclarecer las causas, las condiciones, y las estructuras, aportando a una historia total" (pág. 5). Ello le otorga mayor credibilidad a lo descubierto.

A través del contacto con las personas, Gatica (2010j), considera haber: "rescatado prácticas, percepciones, y discursos; develando la importancia del trabajo como factor organizador y estabilizador, al dotar a estos sujetos, de un sitio en la sociedad receptora, y permitiéndoles "ir desarmando sus valijas", es decir, ir superando la transitoriedad" (pág. 5). Este asunto en concreto, bien podría ser objeto de un estudio motivado por las recientes migraciones de venezolanos hacia el exterior, como consecuencia de la situación vivida en el país. Sin embargo, es necesario aclarar que no es la intención asumirlo en el desarrollo de nuestro proyecto actual.

La aplicación del análisis de red -en consideración a la autora-, facilitó que estas personas se hicieran visibles, transformándolos en actores racionales, con objetivos, que se movilizaron gracias a su disponibilidad de recursos, tratando de superar la creencia o percepción que los convertía en seres desesperados. Por ello, Gatica (2010k), indicó que se trataba de: “[...] mujeres y hombres que eligieron un destino posible, siempre atendiendo al 
doble carácter de la experiencia en que estuvieron inmersos [...]" (págs. 5-6).

La salida de su país les dio la oportunidad de ser liberados, pero también los privó de otros aspectos valorados en sus vidas, pues para Gatica (2010I), esto ocurre: "[...] asociando indisolublemente sentimientos de dolor por el desprendimiento de lo propio -de los afectos personales y colectivos-, agravados por los efectos de la derrota y la incertidumbre" (pág. 6).

También la investigadora manifiesta su propósito de lograr que este trabajo, no sea sólo un estudio de caso, sino que pueda incluirse a partir de su especificidad, dentro de una perspectiva comparativa, que supere la mera definición espacial, es decir, el Noreste de Chubut. Asimismo, para justificar su actividad la autora expresa que, al indagar en la memoria de estos trabajadores, acudió a la perspectiva de Martorell (2001); y Guelerman (2001), citados por Gatica (2010m), asevera que su estudio no se inscribe en:

[...] El ejercicio de la memoria en el sentido de conservar sin modificación, sino de situar lo recordado en el presente para develar su significación actual y propiciar la emergencia de lo nuevo, desde una interrogación que habilite la posibilidad de una crítica histórica. En la reapropiación del pasado, el presente se transforma (pág. 6).

La cita resulta de interés pues sugiere la necesidad de revisar fuentes de distinta naturaleza, que nos aproximen hacia la realidad concreta representada en las caricaturas, como parte de una dialéctica que conducirá a la síntesis del nuevo conocimiento. De esta manera, los resultados de Gatica (2010n), le permitieron enterar: "[...] la dimensión teórica que encierra la memoria para la historiografía [...]" (pág. 6); insertos en los procesos vividos en el Cono Sur, considerando la importancia del trasvasamiento generacional, es decir, la transferencia de las experiencias de padres a hijos, como parte del patrimonio familiar e incluso acervo cultural de la comunidad en estudio. 


\subsection{El humor gráfico}

Este trabajo comienza advirtiendo que si en general, el acercamiento a cualquier tema de investigación requiere una justificación, para Meléndez (2005a), podría parecer que: “[...] estudiar el humor gráfico como contenido periodístico desde una perspectiva histórica necesite una doble argumentación" (pág. 7). No obstante -continúa diciendo-, se observa que la consulta de los diarios u otros medios de comunicación impresos, como punto de partida para el estudio de lo contemporáneo, se hace más frecuente.

Por otra parte, indica la autora, se debe considerar que el acercamiento a dicho material, no representa el acceso a un origen inequívoco de datos, sino a una expresión de estados de opinión. En consecuencia, se deduce que el humor gráfico, siendo parte del mensaje periodístico, conforme a Meléndez (2005b): "[...] es una de las formas más directas, más asimilables para un núcleo amplio de lectores, de expresión de opiniones" (pág. 7). Ambas citas refieren la importancia que le atribuye la autora al hecho de asimilar el humor gráfico por su carácter histórico, aunque deba ser justificada tal condición, dado que esa no es su naturaleza. La misma autora reconoce como virtud de este género periodístico de opinión, el hecho de ser más accesible o asimilable para un segmento mayor de lectores.

Sobre las peculiaridades en las que Meléndez (2005c), llama la atención, reitera que: "[...] el humor gráfico actúa igualmente como testigo de los hechos sobre los que se pronuncia y como elemento que contribuye a la intervención de los medios en el curso de los propios acontecimientos" (pág. 7). Ésta cita reafirma la condición de que el mensaje de la caricatura política tiene tras de sí, la participación testimonial de su autor, quien plasma su opinión sobre los hechos que le rodean, quedando de parte del lector la indagación a profundidad (si fuese de su interés), y la posterior interpretación sobre lo expresado.

Reconoce Meléndez (2005d), que si bien el humor gráfico es un 
modesto aporte: "sobre todo como apoyo en la orientación de la percepción de la realidad, en la construcción social de determinadas concepciones" (págs. 78); las expresiones vertidas en estos particulares espacios periodísticos, por el sólo hecho de asumir situaciones para distinguirlas por encima de otras, y por la manera en que se ofrecen a la audiencia -emitiendo juicios, proyectando valores, actitudes o ideas-, pueden influir en la evolución de esos acontecimientos, pues poco a poco inciden, transformándolo. Las ideas expuestas son argumentos en los que coincidimos para valorar el interés suscitado por el uso de las caricaturas políticas (siendo parte de lo que simboliza el humor gráfico en su más amplio sentido), como foco central en la construcción didáctica asumida en nuestro trabajo.

Explica Meléndez (2005e), que es necesario profundizar en la conexión entre periodismo y humor gráfico: "la vinculación es innegable, toda vez que el desarrollo del primero va a suponer el soporte sobre el que evolucione el segundo" (pág. 8). Sin embargo, a pesar de haber encontrado un espacio para sí en el ámbito periodístico, que le es propio por su enorme desarrollo, el humor gráfico aún no alcanza un lugar prominente como campo u objeto de estudio. Seguidamente, Meléndez (2005f), atribuye esta condición a que quizá su heterogenia: "[...] sea la clave para explicar una cierta intermitencia en el interés académico [...]" (pág. 8); siendo que tal riqueza y diversidad justifique que el humor gráfico habite en: "[...] una tierra de nadie desde la que distintas disciplinas lo acogen a veces sin reclamarlo definitivamente para sí [...]" (pág. 8).

Para afianzar sus observaciones, Lethève (1986), citado por Meléndez (2005g): apoyándose en que él ha enumerado algunas aplicaciones académicas y de investigación en las cuales la ilustración humorística ha sido de utilidad, muchas de ellas ligadas a la estética -por la influencia de las corrientes artísticas sobre el dibujo-; también en la historia de las costumbres -a las que interpreta con fidelidad o de manera distorsionada-; o con los temas 
de mayor interés en cada momento histórico, además de la historia de la opinión pública sobre la cual, por su propia naturaleza, influye u orienta.

Otro de los aspectos destacados por Meléndez (2005h), que capta atención para nuestro trabajo es: "la complejidad que para el analista supone el doble código icónico y verbal del humor gráfico -a la que se suman los laberínticos procesos del fenómeno humorístico-, como contenido del periódico [...]" (pág. 8); lo cual nos alerta sobre la necesidad de incluir entre nuestros objetivos específicos, el diseño de herramientas para descomponer la caricatura, analizarla tanto en su dimensión icónica como verbal, y así develar con certeza el mensaje del autor, contribuyendo a la interpretación.

Esta característica propia del humor gráfico es realzada por Meléndez (2005i), quien detalla que: "[...] este material consigue potenciar esa influencia sobre su época, al gozar de cierta ventaja sobre los casi hegemónicos mensajes textuales de la prensa [...]" (pág. 8); pues tal como la autora reconoce, ésta forma de expresión hace la comprensión más accesible para el público: "[...] aportando símbolos y referentes que pueden formar parte de la mentalidad social, permitiendo incluso desvelar aspectos del pasado que no transmiten las fuentes escritas" (pág. 8). Basado en tales apreciaciones resulta claro para nuestro interés, que dichas características pueden ser claves en la facilitación del proceso de educativo, para el docente y para el alumno.

Uno de los valores atribuidos a las viñetas periodísticas (si bien el término se aplica a un conjunto de recuadros en serie -llamados cartoons 0 comics-, también se puede incluir las caricaturas políticas), para Meléndez (2005j), está centrado el hecho de: "[...] constituir índices de las representaciones que vastos sectores de lectores de prensa se hicieron de los grandes condicionantes, especialmente políticos, de sus vidas, una vía privilegiada de acceso a un repertorio de significados inadvertidos 0 inconscientes" (págs. 8-9).

Tras esta afirmación, Meléndez (2005k), revela que: "resulta lógico que 
nos preguntemos si realmente podemos sacar conclusiones sobre la representatividad de las cuestiones que plantea el humor gráfico como parte de la mentalidad de sus receptores a través del estudio de los propios mensajes" (pág. 9). Profundizando sobre el tema y siguiendo las ideas de Peñamarín (2002), citado por Meléndez (2005I): "la comunicación del humor gráfico se basa en una "complicidad irónica", al darse por supuesto que entre autor y receptor existe un mundo de sentido y valor compartido" (pág. 9).

La autora considera que es sobre esa base que el lector, establece la conexión con el artista, apoyado en sus propias vivencias de los hechos acontecidos, más la suma de su acervo cultural, dando cabida a la satisfacción que genera y da sentido al chiste contenido en la caricatura. De allí la utilidad del humor gráfico para escudriñar los elementos que, en el pasado, fueron parte de la cotidianidad de las personas y la sociedad, constituida a partir de la complicidad que existe entre el artista y su público. Reafirmando lo dicho, Meléndez (2005m), comenta que: “[...] Freud advirtió la gratificación que produce un chiste que nos ofrece un reencuentro donde esperábamos hallar algo nuevo, señalando así la importancia del "factor actualidad”" (pág. 9).

Sobre lo expuesto, asumimos como propias las inquietudes de esta autora cuando certifica que hasta ahora se ha alimentado, casi como tópico, esa concepción de que el humor es algo más serio de lo que parece -así como lo han sostenido nuestros propios humoristas, entre ellos Zapata-, y por extensión, que el humor gráfico es un espacio comprometido y crítico de nuestros periódicos. Tal propósito, se resume a juzgar por Meléndez (2005n), en que:

Estamos de acuerdo en sostener que el humor gráfico es un elemento más entre los posibles discursos que contribuyen al asentamiento o al cambio de valores sociales, pero hasta el momento estas afirmaciones se hacían a la ligera sin someter a un material determinado a un estudio de campo que permitiese el análisis del contenido, de las opiniones vertidas por humoristas gráficos periodísticos, de ahí el interés por 
someter a este contenido tan singular del periódico a un estudio más sistematizado (págs. 12-13).

\section{Conclusiones}

Como corolario de este transitar por el Estado del Arte, es necesario enfatizar aquellos aprendizajes adquiridos en esta búsqueda del conocimiento, confrontada por otros autores desde distintas perspectivas en oportunidades precedentes, y puesto a disposición de la comunidad de investigadores y la sociedad en general. Se trata de plasmar en estas líneas finales, lo que en nuestro criterio constituyen sus principales aportes para el desarrollo ulterior de una Didáctica de la Historia Reciente como constructo dialécticointerpretativo de las caricaturas políticas de Zapata, que constituye la razón de ser en nuestro proyecto de tesis doctoral. Dichos resultados son los siguientes:

- El valor del currículo como soporte esencial y necesario del proceso educativo, y el papel que desempeña el docente para asegurar el cambio educativo implícito en las transformaciones curriculares, teniendo a la didáctica como el vaso comunicante entre la teoría y la praxis, concretada en la cotidiana interacción entre docentes y estudiantes en el espacio del aula, en su más amplio sentido.

- Identificación de la necesidad de incentivar la creatividad, vista como una cualidad que es propia e inherente al quehacer humano, y que como tal tiene el potencial de ser desarrollada a través de la práctica para que se consolide, en beneficio de la formación integral de los docentes y educandos en el proceso de enseñanza aprendizaje.

- Conocimiento de la problemática en el proceso de enseñanza aprendizaje de la Historia, y la valorización de la perspectiva de alumno en esta dinámica, más el reforzamiento del rol mediador del docente.

- Formas de abordaje de los asuntos de interés y objetos de estudio en la perspectiva de la Historia Reciente, a partir del reconocimiento de la 
dimensión teórica de la memoria en la reconstrucción de los hechos históricos.

- La sistematización del estudio del humor gráfico, como fuente testimonial con valor para la historiografía.

\section{Referencias}

Caldera, P. (2020). Pentagrama Didáctico: Una Aproximación Praxiológica de la Gestión del Conocimiento. Revista Scientific, 5(15), 384-404, eISSN: 2542-2987. Recuperado de:

https://doi.org/10.29394/Scientific.issn.2542-2987.2020.5.15.20.384$\underline{404}$

Cázares, L., Christen, M., Jaramillo, E., Villaseñor, L., \& Zamudio, L. (1990). Técnicas actuales de investigación documental. 3ra. edición (Reimpresión 2007), ISBN: 968-24-3829-2. México, D.F.: Editorial Trillas, S.A. de C.V.

Gatica, M. (2010a,b,c,d,e,f,g,h,i,j,k,l,m,n). ¿Exilio, migración, destierro? Los trabajadores chilenos que se asentaron en el Noreste de Chubut a partir de septiembre de 1973. Memorias, historias e implicancias. Tesis Doctoral. La Plata. Argentina: Facultad de Humanidades y Ciencias de la Educación de la Universidad Nacional de La Plata. Recuperada de:

http://www.memoria.fahce.unlp.edu.ar/tesis/te.377/te.377.pdf

Giraldo, G. (2012a,b,c). Didáctica Universitaria: Del Currículo a las Prácticas de Enseñanza. Tesis Doctoral. Colombia: Facultad de Educación de la Universidad de Antioquia. Recuperado de: http://bibliotecadigital.udea.edu.co/bitstream/10495/6941/1/GloriaGiral do 2012 curriculoense\%C3\%B1anza.pdf

Meléndez, N. (2005a,b,c,d,e,f,g,h,i,j,k,l,m,n). El humor gráfico en el diario 
"EI País" durante la transición política española (1976-1978). Tesis Doctoral. Málaga, España: Facultad de Ciencias de la Comunicación de la Universidad de Málaga. Recuperado de:

http://hdl.handle.net/10630/5170

Millán, A. (2008a,b,c,d). La historia que se aprende en la escuela básica venezolana. Percepción y conocimientos del alumnado. Tesis Doctoral. España: Universidad de Barcelona. Recuperada de: http://diposit.ub.edu/dspace/bitstream/2445/41454/1/AML TESIS.pdf

Ortega, H. (2014a,b,c,d,e,f). La creatividad en la enseñanza del docente universitario de la Universidad Autónoma de Zacatecas, México. Tesis Doctoral. Madrid, España: Universidad Complutense de Madrid. Recuperado de: http://eprints.ucm.es/28480/1/T35768.pdf

Rajiad, B., Figueroa, C., \& López, M. (2010). Temas y procesos de la historia reciente en América Latina. ISBN 978-956-8114-87-9. Santiago, Chile: Universidad de Arte y Ciencias Sociales / ARCIS.

Ríos, P. (2006). Psicología. La Aventura de Conocernos. 2da. Edición, ISBN: 978-980-12-1729-9. Venezuela: Editorial Cognitus, C.A. 


\section{Alexis Antonio González Natera \\ e-mail: alangperiodista17@gmail.com}

Nacido en Caracas, Distrito Capital, Venezuela, el 17 de

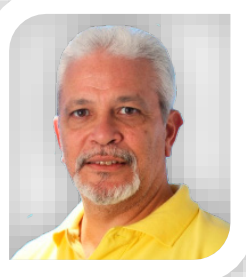
mayo del año 1961. Licenciado en Ciencias y Artes Militares, egresado de la Academia Militar de Venezuela en el año 1985; Especialización en Planificación Global por el Instituto Venezolano de Planificación (IVEPLAN); Maestría en Gerencia Logística por el Instituto Universitario Politécnico de las Fuerzas Armadas Nacionales (IUPFAN); Licenciatura en Comunicación Social, mención honorífica Magna Cum Laude en la Universidad Católica Santa Rosa (UCSAR); Autor del libro "Estrategias logísticas para optimar la gestión de seguridad bancaria" en la Editorial Académica Española (EAE), con el ISBN: 978-3-84735359-1; Ponente invitado en Congresos Nacionales e Internacionales; Docente en Comunicación Social de la Universidad Santa María (USM); Productor de Radio y Locutor Comercial.

El contenido de este manuscrito se difunde bajo una Licencia de Creative Commons ReconocimientoNoComercial-Compartirlgual 4.0 Internacional 